\title{
Direct Comparison between Rotational and Extrusion Rheometers
}

\author{
Asad Ullah Khan, Nasir Mahmood*, Aqeel Ahemd Bazmi \\ Department of Chemical Engineering - COMSATS, Institute of Information Technology, \\ Defence Road, Off Raiwind Road, Lahore, Pakistan 54000
}

Received: July 10, 2009; Revised: October 31, 2009

\begin{abstract}
The use of cone and plate rotational rheology to characterize the flow properties of concentrated dispersion and extrusion methods to determine the rheological properties of pastes have become established rheometric techniques. Direct comparison between the results obtained from the two techniques has not previously been demonstrated. In this article the results of such a comparison using established experimental methods and associated analysis are reported. It is found that for most samples investigated the difference in the calculated values of shear stress at a shear rate of $5 / \mathrm{s}$ is $\leq 10 \%$. Data for a series of slurries, composed of various solid fractions of lime waste, are reported over a shear rate of 0-10/s. The computed results for two techniques are shown to be, within the experimental error, identical over this shear rate range.
\end{abstract}

Keywords: rheology, extrusion, flow, stresses, mechanical properties

\section{Introduction}

Rheology is the science of studying the flow and deformation of materials rooted in the laws of elasticity and viscosity proposed by Hooke and Newton in the late $17^{\text {th }}$ Century. Flow behavior can be conveniently separated into three components: Shear and extensional flows which are characterized by the corresponding viscosities and Elastic behavior which is characterized by measurement of modulus or swell ratios. Proper characterization of flow behavior is likely to require sophisticated and versatile instrumentation. To fully characterize a material, instrumentation is required which has the capability of extracting these parameters over a range of temperatures and shear/extension rates. Modern laboratory rheological test apparatus can be divided into two broad categories of rotational rheometers and capillary extrusion rheometers.

Extrusion (capillary and orifice) is a well established methodology which is adopted in the processing of relatively soft solids. This technique and the associated analysis were developed for the processing of polymer melts and solutions, but the same principle has been used in ceramics and food processing as well as in the allied industries ${ }^{1-8}$. Extrusion is used in the ceramic industry for the manufacturing of different shapes ranging from very simple geometries such as clay bricks to very complicated articles such as ceramic honey comb matrices used in a car exhaust system to control the exhaust emissions? Extrusion is also used in the metal industry for production of articles such as wires and rods. Various analytical treatments which have been adopted, based upon metal plasticity approaches using extrusion, may be found in the literature ${ }^{10-12}$. These theories have been modified so that they may be applied to ceramic pastes ${ }^{2,13}$. The main modification is the introduction of a velocity dependent flow stress term into the equation for the computation of the extrusion pressure. The capillary extrusion flow experiment has also been used to measure the boundary slip velocity between the capillary wall and the flowing material ${ }^{2} 14$. The rheological properties of complex fluids are greatly influenced by the interface between the wall of rigid equipment and the material. The boundary condition has a significant effect upon the experimental results obtained. During shear, the energy is transmitted from the rigid walls of operating equipment to the bulk of material. Knowledge of boundary conditions between the walls and the material is necessary in order to deconvolute the true rheological characteristics of the material under investigation. In addition, during the course of the imposed deformation and flow, the boundary between the material and the wall develops its own characteristics.

A more conventional means of determining the rheology of such materials, although it can not be used for very high volume fraction plastic fluids, is the rotational cone and plate rheometer system. One of the advantages of using these instruments is that the shear rate is nominally independent of the position of the fluid within the shear zone, provided the cone angle is very small $\left(\leq 5^{\circ}\right)^{15}$. Rotational rheometers are the preferred choice when the requirement is to obtain information concerning the molecular structure and how this affects processing characteristics. In particular, the ability to easily extract information about the average molecular weight and molecular weight distribution via measurement of the viscoelastic properties makes the rotational rheometer a powerful tool. The capillary rheometer extends the shear rate range attainable in the laboratory beyond that available in a rotational instrument and allows the flow properties to be measured under typical processing conditions.

Although the capillary extrusion and the cone and plate rheometers have been extensively used independently, direct comparison of the results provided by these two techniques is rarely undertaken. One of the reasons for this may be due to the fact that the capillary extrusion method is normally used for relatively concentrated suspension/pastes while the cone and plate technique is adopted for relatively less concentrated suspensions. Generally, a given system is not amenable to an evaluation by using of both techniques within similar compositional ranges. The material used in this study is shown to be appropriate for the study by both techniques thus allowing a direct comparison of the results obtained by the both techniques.

The capillary extrusion and cone and plate rheometers have different flow geometries. Despite the different geometries involved in capillary and cone and plate rheometers, a comparison of the data is possible, since the data may, in principle be reduced to similar material response parameters. In both cases certain assumptions 
have to be introduced in order to obtain these parameters. These assumptions are:

- The material is incompressible;

- The flow behavior of the material is not a function of lapsed time, and is laminar and steady in character;

- There is no slip involved between the capillary wall (and plate surface) and the material extruded;

- The end effects are negligible; and

- In addition, for the case of extrusion, the viscosity of the fluid remains constant within the capillary and is unaffected by the changes in hydrostatic pressure component along the capillary length.

\section{Analysis}

\subsection{Capillary extrusion analysis}

A capillary rheometer consists of a ram and a cylinder. The ram moves inside a cylinder which contains the samples to be extruded. At the other end of the cylinder, a capillary is attached. When the ram moves, the material flows through the capillary. A schematic diagram of a capillary rheometer (extruder) is given in Figure 1. The apparent shear rate, $\dot{\gamma}_{\mathrm{w}}$ along the walls of the capillary is given by Equation (1).

$$
\dot{\gamma}_{w}=-\frac{4 Q}{\pi R^{3}}
$$

where $\mathrm{Q}$ is the volumetric flow rate, which can be calculated from Equation (2)

$$
Q=\pi R_{r}^{2} V_{r}
$$

where $R_{r}$ is the radius of the ram and $V_{r}$ is the velocity of the ram.

The shear stress at the walls of the capillary is calculated from following Equation (3).

$$
\tau_{w}=\frac{R \cdot \Delta P}{2 L}=\frac{D \cdot \Delta P}{4 L}
$$

where $\Delta \mathrm{P}$ is the net resulting pressure required to extrude the material through a capillary of length $\mathrm{L}$.

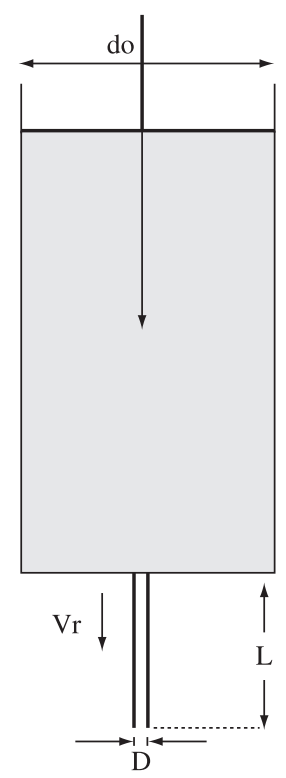

Figure 1. Capillary extrusion; $D=2 \mathrm{R}, \mathrm{Vr}$ is the velocity of the ram, $\mathrm{do}=2 \mathrm{Rr}$ and $\mathrm{L}$ is the length of the capillary.
Equations (1) and (3) were used to calculate the shear rate and the shear stress, respectively, for the capillary extrusion experiments.

\subsection{Cone and plate viscometer: Analysis}

A schematic diagram of the cone and plate viscometer assembly is shown in Figure 2. If the gap angle, $\theta$ is small $\left(\leq 5^{\circ}\right)$, the shear rate is approximately constant everywhere within the sample ${ }^{15}$ and is given by Equation (4).

$$
\dot{\gamma}=\frac{\Omega}{\theta}
$$

where $\Omega$ is the angular velocity of rotating plate.

The shear stress, $\tau$, on the cone is given by Equation (5):

$$
\tau=\frac{3 C}{2 \pi r^{2}}
$$

where $r$ is the radius of the cone and $\mathrm{C}$ is a constant which depends upon the characteristics of the instrument. In the Bohlin VOR rheometer used in this work, the system software is calibrated by the manufacturer to calculate, $\mathrm{C}$, when the cone and plate assembly is used. The data obtained from the rheometer is in the form of shear stress as a function of shear rate, and thus eliminating the need of knowing the value of "C" separately.

\section{Experiments}

\subsection{Materials}

The material used in these experiments was a soda ash waste containing high natural moisture content, between $55-70 \%(\mathrm{w} / \mathrm{w})$. The moisture appeared to be encapsulated within a silt-sized cellular structure. The main constituents were calcium sulphate and calcium chloride with varying amounts of water along with other impurities. The $\mathrm{pH}$ values of the samples used varied from 10.1 to 11.0. The material was extracted from a waste site in the United Kingdom. Four $10-15 \mathrm{~kg}$ samples were extracted from different parts of the site. The samples were stored in plastic bags and thoroughly mixed using a Z-blade mixer before use.

The solid content of the soda ash waste was particulate in nature with a particle size distribution which is summarized in Table 1. $\mathrm{D}(\mathrm{v}, 0.9), \mathrm{D}(\mathrm{v}, 0.5)$ and $\mathrm{D}(\mathrm{v}, 0.1)$ are the average diameters of the particles of volume fraction $0.9,0.5$ and 0.1 , respectively.

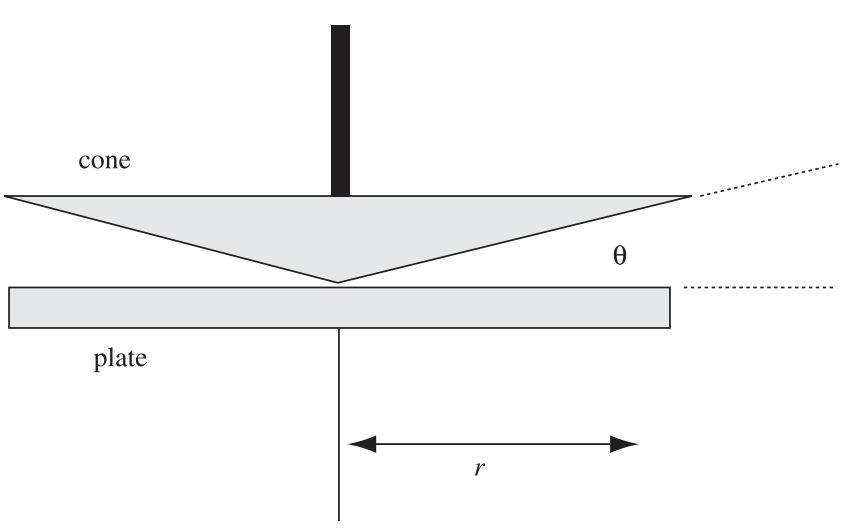

Figure 2. Schematic diagram of the cone and plate rheometer. $\theta$, is the gap angle between cone and plate and $r$, is the radius of the cone (and plate as well). 
Table 1. Summery of the particle size $(\mu \mathrm{m})$ distribution (diameter) of different lime samples.

\begin{tabular}{cccc}
\hline Sample $\mathrm{n}^{\mathrm{o}}$. & $\mathrm{D}(\mathrm{v}, 0.1)$ & $\mathrm{D}(\mathrm{v}, 0.5)$ & $\mathrm{D}(\mathrm{v}, 0.9)$ \\
\hline 1 & 2.0 & 4.5 & 7.9 \\
2 & 2.7 & 5.6 & 9.6 \\
3 & 2.6 & 5.0 & 9.0 \\
4 & 2.8 & 6.2 & 10.2 \\
\hline
\end{tabular}

\subsection{Cone and plate rheometer}

A "Bohlin VOR Rheometer" was used with a cone and plate assembly designed as " $\mathrm{CP} 5 / 30$ " to measure the shear stress as a function of shear rate. All the experiments were carried out at room temperature (ca. $20^{\circ} \mathrm{C}$ ).

\subsection{Capillary extrusion}

The apparatus used for extrusion is shown schematically in Figure 1. This apparatus consisted of a rigid cylindrical ram with smooth surface, which was moved at a constant (but adjustable) velocity in a rigid cylinder of diameter $20 \mathrm{~mm}$ in order to extrude the sample through an orifice or a capillary (or die-land). An orifice is regarded as a capillary with negligible length. In the apparatus used, variables orifices of different diameters ( 1 to $4 \mathrm{~mm}$ ) and capillaries of different diameters (1 to $4 \mathrm{~mm}$ ) and lengths $(20$ and $30 \mathrm{~cm}$ ) were used. During extrusion, the ram was displaced by an Instron Machine 6022 cross-heated upon which a load cell was mounted. This load cell was connected to a computer to record the force/displacement data (reaction load vs. time). The load values were converted to shear stress by Equation (3) and the ram velocity and ram movement was converted to a shear rate by Equations (1) and (2).

\section{Results and Discussion}

The data shown in Figure 3 to 6 for the cone and plate rheometer were obtained in shear rate increasing (up) and decreasing (down) modes. It is seen that in each figure, the data are almost identical in the "up" and "down" modes at shear rates greater than ca.2/s. Below a $(2 / s)$ shear rate value the data are very scattered and, in general, shear stress is higher when the shear rate is increasing (up mode) than that of when the shear rate is decreasing (down mode). When the shear rate commences to increase from rest, the shear stress also increases

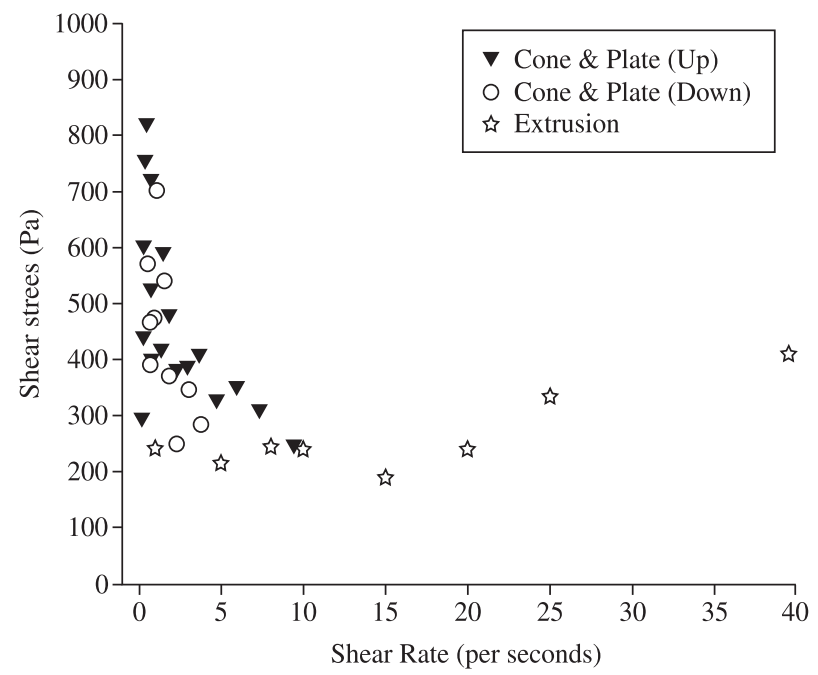

Figure 3. Shear stress as a function of shear rate of sample 1, measured using cone and plate rheometer and capillary extrusion.

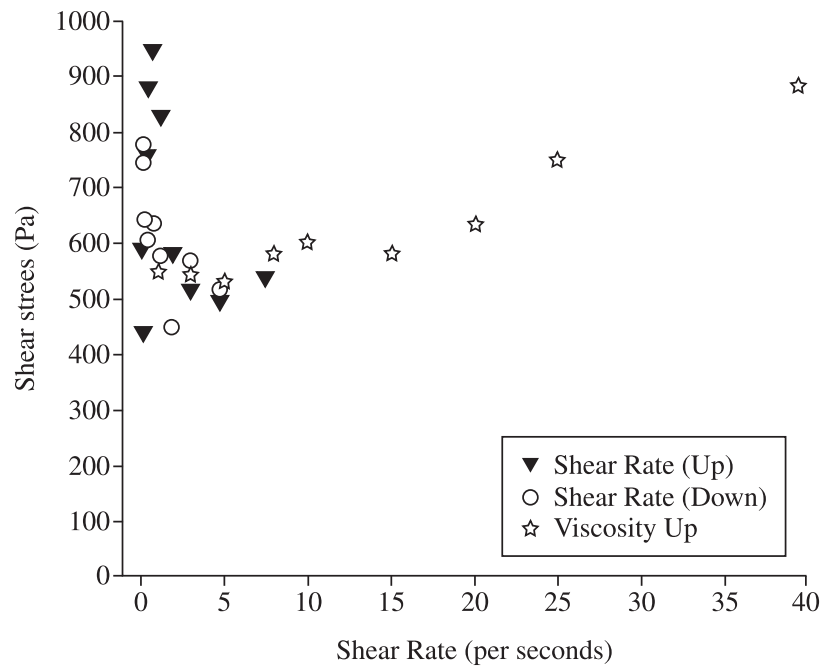

Figure 4. Shear stress as a function of shear rate of sample 2, measured using cone and plate rheometer and capillary extrusion.

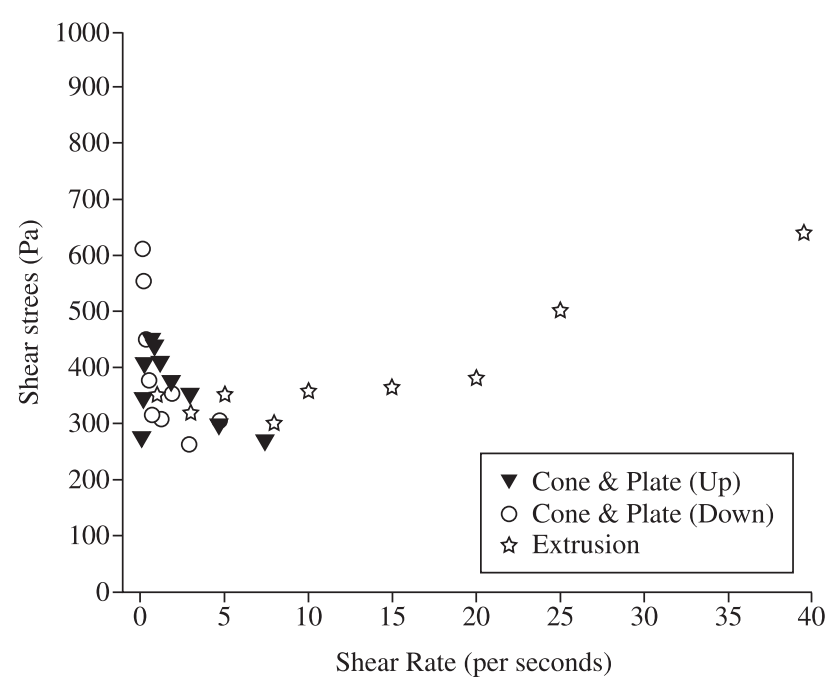

Figure 5. Shear stress as a function of shear rate of sample 3 , measured using cone and plate rheometer and capillary extrusion.

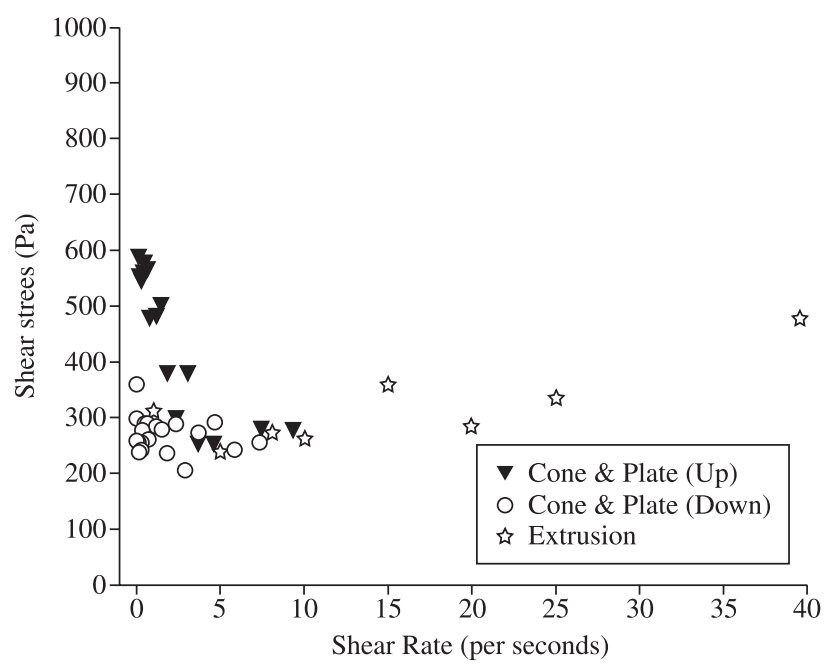

Figure 6. Shear stress as a function of shear rate of sample 4, measured using cone and plate rheometer and capillary extrusion. 
and shows a maximum, and thereafter starts decreasing and reaches a virtually constant value at shear rates greater than ca.2/s. This is almost certainly due to the fact that when the shear rate starts increasing from the rest, the "bonding" and structural arrangement (e.g. alignment of particles) between the particles of the material begins to deform. When the shear stress reaches a maximum the "bonds" between the particles begin to break down, and thereafter there is a steady decrease in the shear stresses. A similar maximum has been observed by many other researchers ${ }^{16-18}$. The same argument also explains why the shear stress is higher when the shear rate is decreasing. When the shear rate is an increasing function, the structural changes due to stretching and bending of bonds occur, while when the shear rate is decreasing, the bonds which had been previously disrupted do not immediately reform within the time scale of the experiment. The induced structural changes also cause the moisture entrapped within the structure to be released. Therefore, the maximum observed in the shear stress value during the shear rate increasing mode is greater than the value that is observed for another maximum during the shear rate decreasing process.

In contrast, the data obtained for the capillary extrusion (Figure 3 to 6) show no maxima in the shear stress against shear rate. This behavior may be attributed to the non-steady shear rate increase imposed in the capillary extrusion experiment. In the case of the cone and plate viscometer configuration the shear rate was increased steadily in small increment while in the case of extrusion the shear rate increase was non-steady and the step increase involved were much larger than those adapted for the cone and plate rheometer studies. In the case of cone and plate rheometer, the shear rate was changing without stopping the rotation of the rheometer, where as for the case of the capillary extrusion, the extrusion process was stopped for each change in the shear rate. Since the shear rate increase involved was non-steady, there was no progressive stretching and deformation of the bonds between particles (structural changes) involved for the case of capillary extrusion.

Comparing the cone and plate rheometer data with capillary extrusion for sample 1 in Figure 3, the data show a computed shear stress between ca. 250-350 Pa. for the shear rate between 2 and 10/s. For the same shear rate range, using capillary extrusion, the shear stress values obtained lie between ca. 200 and $250 \mathrm{~Pa}$.

Figure 4 compares the data for sample 2 for the two rheometers. The Figure indicates that the shear stress varies between ca. 450 and $600 \mathrm{~Pa}$ for the shear stress rates between 2 and 8/s for the cone and plate rheometer. In case of the capillary extrusion for the same material (sample 2), it may be seen that the shear stress shown in Figure 4 is virtually constant for the shear rate between 2 and $8 / \mathrm{s}$ and is ca $550 \mathrm{~Pa}$. This computed value is very comparable with the data obtained using cone and plate rheometery system.

Figure 5 gives shear stress vs. shear rate plots for the two rheometers being compared for sample 3 . For the cone and plate rheometer, the shear stress was found to lie between 260 and $360 \mathrm{~Pa}$, for the shear rate range between 2 and 8/s. In the same shear rate range, the capillary extrusion data indicate the shear stress lies between ca. 300 and $370 \mathrm{~Pa}$. Figure 6 provides the shear stress- shear rate relationships for sample 4 . The data, which were obtained using the cone and plate rheometery, indicate that the shear stress lies between ca. 250 and $300 \mathrm{~Pa}$ (except at shear rate ca.3/s) for the shear rate range between 2 and 10/s. Using capillary extrusion method for the same shear rate range, the shear stress which was calculated ranged between 250 and $300 \mathrm{~Pa}$.

The capillary extrusion results in Figure 3 to 6 show two types of shear stress-shear rate behavior for all the samples; one below shear rate ca.15/s and the other above a shear rate condition ca.15/s. Below the shear rate of ca.15/s the shear stress is virtually constant
Table 2. Maximum difference/error in shear stresses (Pa) at Shear Rate 5/s of different samples.

\begin{tabular}{ccccc}
\hline $\begin{array}{c}\text { Sample } \\
\text { No. }\end{array}$ & $\begin{array}{c}\text { Cone \& } \\
\text { Plate }\end{array}$ & Extrusion & Difference & $\begin{array}{c}\text { Relative } \\
\text { Difference \% }\end{array}$ \\
\hline 1 & 330 & 230 & 100 & 30 \\
2 & 500 & 530 & 30 & 6 \\
3 & 300 & 330 & 30 & 10 \\
4 & 250 & 230 & 20 & 8 \\
\hline
\end{tabular}

and above this shear rate the shear stress increases steadily as the shear rate increases. This phenomenon may be due to the peculiar structure of this material. At shear rate less than $15 / \mathrm{s}$, the material has a structure which has entrapped moisture within it and at higher shear rates $(>15 / \mathrm{s})$ this structure is broken down and thus the material behaves rather differently.

The major feature to be noted here is the similarity in the form of flow curves measured with the cone and plate rheometer and the extrusion rheometer particularly over the shear rate range 1 to $10 / \mathrm{s}$. Moreover, the computed magnitude of the shear stresses is remarkably similar for both cases. This conclusion is best illustrated in Table 2 where the shear stresses for the two methods at a shear rate of $5 / \mathrm{s}$ are given. The computed data vary by no more than $30 \%$ for the case of sample 1 and for the other samples the maximum discrepancy involved is $\leq 10 \%$. Given the assumptions made in the two analyses, the results reported in this paper are encouraging to make comparison of the two rheological techniques.

\section{Conclusions}

Capillary extrusion is used widely to investigate the rheological properties of relatively concentrated suspensions/paste systems. In contrast the cone and plate rheometer arrangement is used for comparatively less concentrated suspensions. For intermediate range suspensions of volume fraction it is possible to use either of these techniques. In the present work it has been demonstrated that for moderately concentrated suspensions the results obtained by the two methods are quite comparable and fall within the range of (their respective) experimental errors. Thus, by combining these two techniques it is possible to determine a number of rheological properties for a wide range of suspensions/pastes.

\section{References}

1. Tadmor $Z$ and Gogos CG. Principles of Polymer Processing. $2^{\text {nd }}$ Edition. New York: Wiley; 2006.

2. Briscoe JB, Khan AU and Luckham FP. Evaluation of slip in ceramic paste extrusion. Journal of the European Ceramic Society. 2001; 21(4): 483-491.

3. Luukkonen P, Newton JM, Podczeck F and Yliruusi J. Use of a capillary rheometer to evaluate the rheological properties of microcrystalline cellulose and silicified microcrystalline cellulose wet masses. International Journal of Pharmaceutics. 2001; 216(1-2):147-157.

4. Forzatti P, Ballardini D and Sighicelli L. Preparation and characterization of extruded monolithic ceramic catalysts. Catalysis Today. 1998; 41(1-3):87-94.

5. Das RN, Madhusoodana CD and Okada K. Rheological studies on cordierite honeycomb extrusion. Journal of the European Ceramic Society. 2002; 22(16):2893-2900.

6. Liang Z and Blackburn S. Design and characterisation of a co-extruder to produce trilayer ceramic tubes semi-continuously. Journal of the European Ceramic Society. 2001; 21(7):883-892. 
7. XU Z and Raphaelides SN. Flow behavior of concentrated starch dispersions using a tube rheometer of novel design. Journal of Texture Studies. 1998; 29(1):1-13.

8. Alfani R, Grizzuti N, Luca Guerrini G and Lezzi G. The use of the capillary rheometer for the rheological evaluation of extrudable cementbased materials. Rheological Acta. 2007; 46(5):703-709.

9. Benbow J and Bridwater J. Paste Flow and Extrusion. Oxford: Claredon Press; 1993.

10. Avitzur B. Metal Forming: Processes and Analysis. New York: McGraw Hill; 1968.

11. Johnson W and Mellor PB. Engineering Plasticity. London: van Nostrand Reinhold Co.; 1973.

12. Rowe GW. Principles of Industrial Metal and Working Processes. London: Edward Arnold; 1977.

13. Benbow JJ, Jazayeri SH and Bridgwater J. The flow of pastes through dies of complicated geometry. Powder Technology. 1991; 65(1-3):393-401.
14. Yilmazar U and Kaylon DM. Slip effects in capillary and parallel disk torsional flows of highly filled suspensions. Journal of Rheology. 1989; 33(8):1197-1212.

15. Barnes HA, Hutton JF and Walter K. An Introduction to Rheology. Amsterdam, The Netherlands: Elsevier Science Publishing B.V.; 1989. p. 30 .

16. Kawaguchi M. Effects of polymer adsorption and desorption on rheological properties of silica suspensions. Colloids and Surfaces A: Physicochemical and Engineering Aspects. 1994; 86 (pp.FM3):283-289.

17. Kawaguchi M, Kimura Y, Tanahashi T, Takeoka J, Suzuki J-ichi, Kato T and Funahashi S. polymer adsorption effects on structures and rheological properties of silica suspensions. Langmuir. 1995; 11(2):563-567.

18. Ryo Y, Nakai Y and Kawaguchi M. Viscoelastic measurements of silica suspensions in aqueous cellulose derivative solutions. Langmuir. 1992; 8(10):2413-2416 